\title{
ANN method for size determination of storage systems in microgrids
}

\author{
Yaser Qudaih*, Thongchart Kerdphol, Yasunori Mitani \\ Department of Electrical and Electronics Engineering, Kyushu Institute of Technology, 1-1, Sensui-cho, Tobata-ku, Kitakyu-shu, \\ Fukuoka, 804-8550, Japan
}

\begin{abstract}
In the recent years, storage systems played significant roles than ever. One of the reasons behind such a phenomenon could be associated with the increase dependency on Renewable Energy Resources (RES) in power systems. Hence, the ability to calculate Battery Energy Storage System (BESS) size for the stand-alone microgrid is very crucial. Artificial Neural Network (ANN) has been proven to be a successful type of Artificial Intelligence (AI) in many applications. This paper presents the design of optimum size of BESS using artificial neural network. The patterns used in the neural network training are sets of frequency and voltage of the stand-alone or isolated system considered as microgrid. The neural network model is developed using simulation data from a nonlinear model. Simulation results show that the proposed neural network can provide an accurate and effective estimation of BESS size for the microgrid. Moreover, the optimal size of BESS-based ANN gives an improved performance than the BESS-based predefined size in the microgrid system.
\end{abstract}

Keywords: Artificial neural network, battery energy storage system, frequency control, microgrid, optimization

\section{Introduction}

The world is in a kind of energy transition. Not only environment issues but economic and social matters are at stake. Technology is still playing the same important role to facilitate the provision of the society needs and requirements. Power system is a vital arrangement for the humanity. The way of developing power systems has to be in the same line with the people's need. However, the demand is dramatically increasing and conventional power generation improvement is a contradictory with the fossil oil depletion and environment concerns. RES are introduced to solve the problem and their integration to the system requires lots of care. On the other hand, energy storage systems became a key issue helps in integrating and utilizing renewable energy in an optimal level.

BESS have been discussed widely in the recent decades. They are considered as an important part of the power system ranging from small applications as in the electronic devices to a bigger size of storage used in power stations and recently to be used as power sources to backup renewable energy. As in [1] optimal sizing of the storage devices has been decided to be closer to the large loads taking into account the voltage stability and loss minimization. However, the presence of RES in the system with storage utilization was almost present in most of the resent researches [2], [3]

On the other hand, EVs with their unique characteristics occupied a remarkable position in the sustainable development, especially in the developed countries. However, the applications of EV do not stand on the mobile vehicle concept transport but go beyond that to be part of the power system. According to the Global EV Outlook report, released in April, 2013, in the worldwide BEV market, Japan holds the largest share due to sales of the Nissan LEAF, followed by the United States, then China and France is in the fourth spot. With applying Inverter, it is possible to deliver electric power from EV

\footnotetext{
${ }^{*}$ Manuscript received April 29, 2015; revised August 10, 2015.

Corresponding author. E-mail address: yaser_qudaih@yahoo.com

doi: $10.12720 /$ sgce.4.3.247-254
} 
battery storage to the facilities [4]-[6].

Artificial intelligence has been also used to solve this matter [7], [8]. Artificial Neural Network (ANN) has been proven to be a successful type of Artificial Intelligence (AI) in many applications. This paper presents the design of optimum size of BESS using artificial neural network. The patterns used in the neural network training are sets of frequency and voltage data of the stand-alone or isolated system which is considered as microgrid.

Section II of this paper discusses the microgrid design and structure. Section III explains the way of utilizing ANN to solve the optimization problem related to the BESS best size based on maintained frequency and voltage. Section IV illustrates the simulation results in the form of graphs and Section V cocludes.

\section{System Configuration}

\subsection{Microgrid}

A typical characteristic of a microgrid is that it can be operated either in grid-connected mode or isolated mode. Under normal operation, the microgrid is connected to the utility grid. Fig. 1 shows the microgrid system which consists of a 1.2 MW mini-hydro generator, $2 \mathrm{MW}$ hydro generator and $3 \mathrm{MW}$ photovoltaic sources. BESS is connected to the microgrid system at bus number 14 . The system also consists of group of feeders which could be part of the distribution system design. The critical loads 1 and 4 require a local generation and the non-critical loads 2, 3 and 5 are not connected to any local generation.

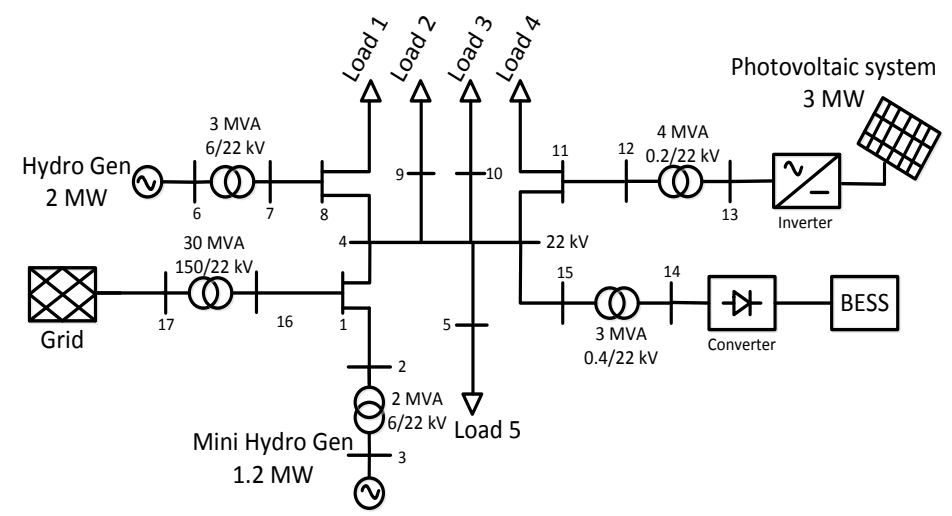

Fig. 1. Microgrid architecture [9].

\subsection{Solar power plant}

The output power of the photovoltaic (PV) is uncertain due to the environmental factors, namely the environmental random changes will inevitably lead to constant changing in the output power of the PV [10], [11]. In order to represent PV characteristic in operating conditions, the influences of solar insolation and atmosphere temperature are designed. The temperature effect is represented by a temperature coefficient of power $T_{c o}\left(1 / \mathrm{c}^{\circ}\right)$. The inverter efficiency is multiplied by the DC output converting DC into AC output as shown in equation (1).

$$
P_{P V}=n_{P V} P_{\text {rate } P V}\left(G / G_{0}\right)\left(1-T_{c o}\left(T_{A}-25^{\circ}\right)\right) \eta_{\text {inv }} \eta_{\text {rel }}
$$

where $n_{P V}$ is the number of PV modules, $P_{\text {rate } P V}$ is the rated PV array electrical power (W), $G$ is the global insolation on the PV array $\left(\mathrm{W} / \mathrm{m}^{2}\right), G_{0}$ is the standard amount of insolation used to rate the capacity of PV modules $\left(\mathrm{W} / \mathrm{m}^{2}\right), T_{A}$ is the ambient temperature, $T_{C O}$ is the temperature coefficient of the maximum power of PV, $\eta_{\text {rel }}$ is the relative efficiency of the PV modules, $\eta_{i n v}$ is the efficiency of the inverter. 


\subsection{Battery Energy Storage System (BESS)}

BESS can be utilized in several implementations such as peak shaving, real power control and load leveling. This paper uses BESS for enhancing the performance of frequency control as BESS can provide active power compensation in a short period of time. Recently, a huge number of electric companies and independent system operators have shown growing interest in BESS due to decreasing cost of batteries. With the fast development of technologies, BESS is expected to be used in several applications including the one proposed in this paper. The structure of BESS consists of power converters, battery cells and control parts which are shown in Fig. 2.

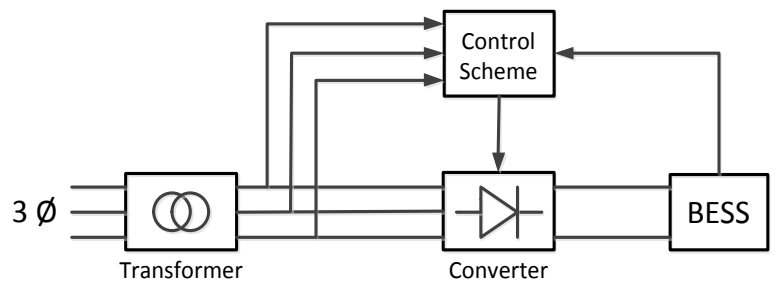

Fig. 2. Structure of BESS and interconnection diagram.

When the power generated by the microgrid system is greater than the load demand, the surplus power can be stored in BESS for future use. On the other hand, when there is any deficiency in the power generation of the microgrid, the stored power can be used to supply the load. Thus, this will improve the system performance and reliability.

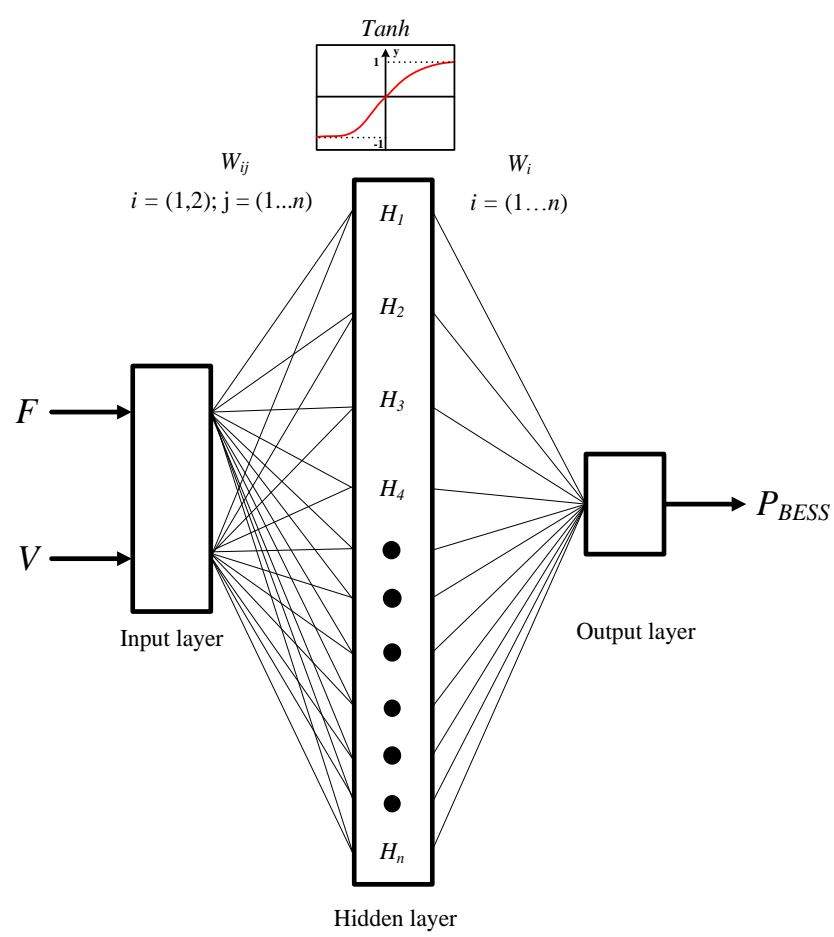

Fig. 3. Three layers architecture of the proposed neural network for BESS size optimization.

\section{BESS Size Optimization Based ANN}

Multilayer perceptron, trained by the standard Back-Propagation (BP) leaning algorithm, is known as 
the feed-forward back-propagation neural network. In this paper, the multilayer perceptron consists of three layer neurons. It shows that one hidden layer is contained and one type of activation function is used in the hidden layer. Hence, this paper can find the optimal size of BESS to supply the microgrid when the microgrid is separated from the main grid based on the measured voltage and frequency of the microgrid. As a nonlinear modelling method, the multilayer perceptron neural network has nonlinearity quality and it can guarantee the accuracy of fitting and there are two inputs with one output in this study as shown in Fig. 3.

where $F$ is the frequency of the microgrid system (Hz.) while $V$ is the voltage of the microgrid system (pu.). $P_{B E S S}$ means the power capacity of BESS (MW).

The output value of the neural network is initiated by transforming a weight total of input based on a transfer function. The transfer function is used to convert the activate level of a neuron to an output value. In this paper, the hyperbolic tangent transfer function (Tanh) is activated between the input layer and the output layer as follow:

$$
y_{i}=f_{\text {Tanh }}\left(x_{i}\right)=\frac{e^{x}-e^{-x}}{e^{x}+e^{-x}}
$$

The hyperbolic tangent activation function is categorized in the logistic type and the output value saturated between -1 to +1 .

First, the output of the hidden layer can be shown as:

$$
\left[\begin{array}{c}
g_{1} \\
g_{2} \\
\bullet \\
g_{i}
\end{array}\right]=f_{\text {Tanh }}\left(\left[\begin{array}{cc}
W_{11}^{(1)} & W_{21}^{(1)} \\
W_{12}^{(1)} & W_{22}^{(1)} \\
\bullet & \bullet \\
\bullet & \bullet \\
W_{1 j}^{(1)} & W_{2 j}^{(1)}
\end{array}\right] \cdot\left[\begin{array}{c}
F \\
V
\end{array}\right]\right)
$$

From (3), it can be simplified as:

$$
g(F, V)=f_{\text {Tanh }}\left(W^{(1)}(F, V)^{T}\right)
$$

The output of the neural network can be expressed as:

$$
P_{\text {BESS }}(F, V)=f_{\text {Tanh }}\left(W^{(2)} g(F, V)\right)
$$

Finally, the proposed neural network model can be expressed as:

$$
P_{B E S S}(F, V)=f_{\text {Tanh }}\left(W^{(2)} f_{\text {Tanh }}\left(W^{(1)}(F, V)^{T}\right)\right)
$$

Table 1. Multilayer perceptron ANN parameters

\begin{tabular}{|l|l|}
\hline Parameters & Multilayer perceptron ANN \\
\hline Goal (MSE) & 0.0001 \\
\hline Inputs & 2 \\
\hline Outputs & 1 \\
\hline Hidden layer & 1 \\
\hline Training data & 30 \\
\hline Testing data & 4 \\
\hline Hidden layer neurons & 10 \\
\hline Output layer neurons & 1 \\
\hline Transfer function & Tanh \\
\hline
\end{tabular}


From Table 1, the parameter details for the proposed neural network are shown and used in training and in the testing database.

Based on the chart in Fig. 4, the outline of the whole process of the proposed neural network is demonstrated. It can be seen that the Mean Square Error (MSE) is compared between desired and actual data where the network will stop when the error is less than 0.0001 .

\section{Simulation Results}

To demonstrate how well the proposed neural network model can achieve the nonlinear relationship between power capacity of BESS $\left(P_{B E S S}\right)$, frequency and voltage of the microgrid, the trained neural network is used to predict the power energy of BESS $\left(P_{B E S S}\right)$ by using the same input data as those in the training data set. In ANN training results, the outputs (the estimated $P_{B E S S}$ ) can be achieved accordingly. As shown in Fig. 5, the corresponding correlation coefficient (R) is 0.99979 which is almost equal to 1. Hence, the proposed neural network model is of high accuracy.

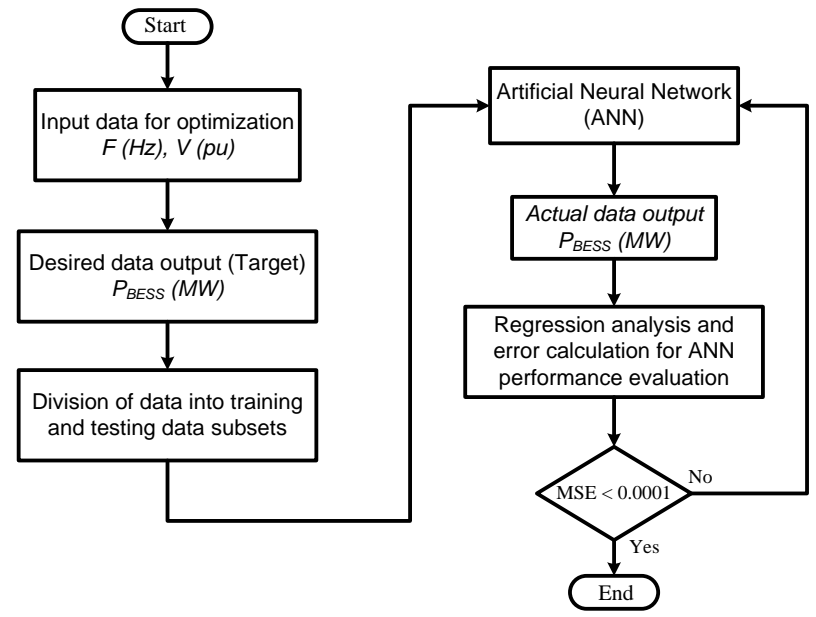

Fig. 4. Summarize of the proposed neural network for predicting BESS size.

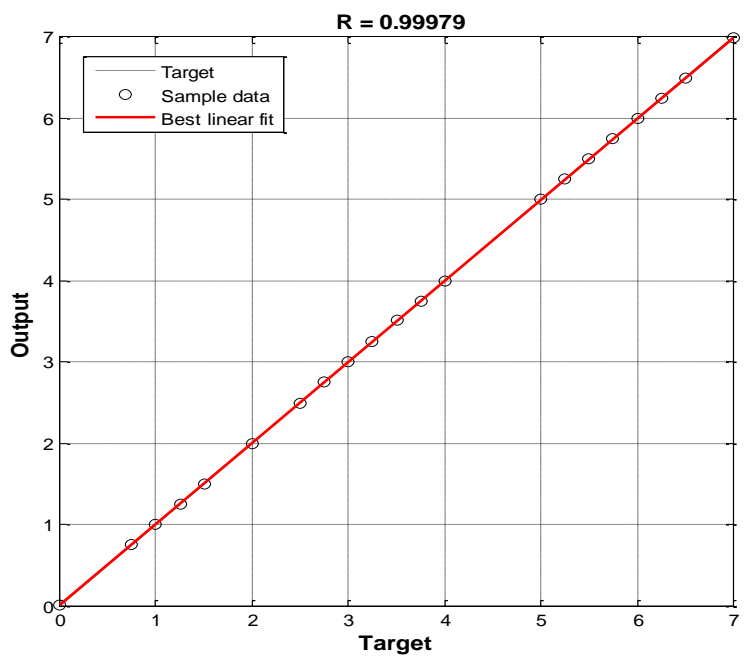

Fig. 5. Regression analysis between estimated output and measured target.

In ANN testing results, to illustrate the accuracy of the proposed neural network approach, the verification process is shown as Fig. 6. 

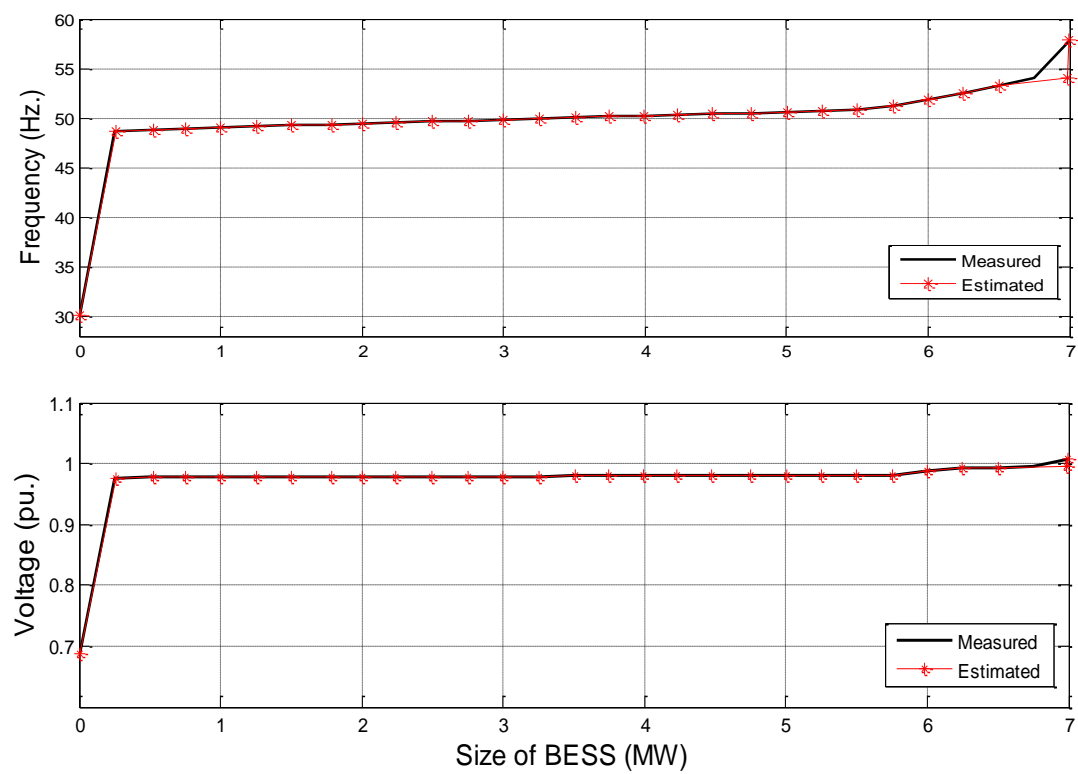

Fig. 6. Comparison between estimated BESS size and measured BESS size based on frequency and voltage of the microgrid.

It can be seen in the above graphs that the estimated BESS size closely follows the pattern of measured BESS size. This confirms that the proposed neural network model is high accuracy.

The mean square error (MSE) on predicting performance shows the learning and generalization error of the normalized valued of BESS size (See Table 2).

Table 2. MSE error for BESS size estimation

\begin{tabular}{|c|c|}
\hline Performance predictor & MSE \\
\hline$P_{B E S S}$ & 0.00000949 \\
\hline
\end{tabular}

This value revealed that the proposed neural network was able to accurately predict the optimum BESS size. MSE equation can be expressed as:

$$
M S E=\sqrt{\sum_{1}^{n}\left(T_{i}-O_{i}\right)^{2}}
$$

where $T_{i}$ is the target vector, $O_{i}$ is the output vector and $\mathrm{n}$ is the number of training data or data for each test in the testing data set.

According to Table 3, the optimal size of BESS is determined by the proposed neural network model based on frequency and voltage of the microgrid.

Table 3. Optimized parameter based on ANN

\begin{tabular}{|c|c|c|}
\hline$P_{B E S S}(\mathrm{MW})$ & $f$ (Hz.) & $v$ (pu.) \\
\hline 3.3488 & 50.000 & 0.979 \\
\hline
\end{tabular}

Looking at Fig. 7, the peak magnitude of the frequency deviation with the BESS-based predefined size reaches $50.3 \mathrm{~Hz}$ and it cannot not recover to the normal state equilibrium (i.e., $50 \mathrm{~Hz}$ ).

In case of no BESS, the system frequency goes out of synchronization because the power supply cannot meet the load demand. It can be seen in that the performance of the system frequency is much 
better when the optimal sizing of BESS-based ANN is considered. It is clearly seen that there is a significant improvement of the frequency performances of the microgrid system in terms of peak deviation and settling time.

Besides, the voltage deviation of the microgrid system is shown in Fig. 8. According to this figure, the optimal sizing of BESS-based ANN can remain the same voltage deviation as the BESS-based predefined size. In case of no BESS, the system voltage dropped drastically because the power supply cannot meet the load demand.

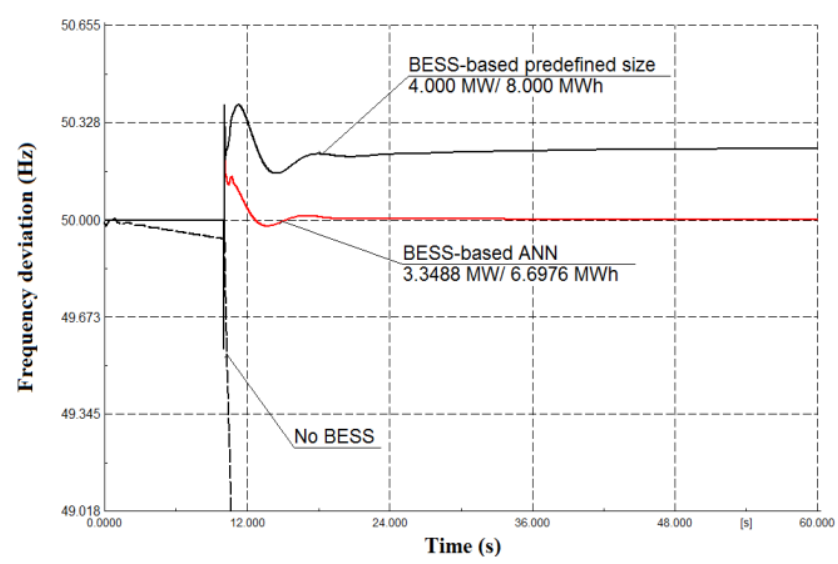

Fig. 7. Frequency deviation of the microgrid after islanding.

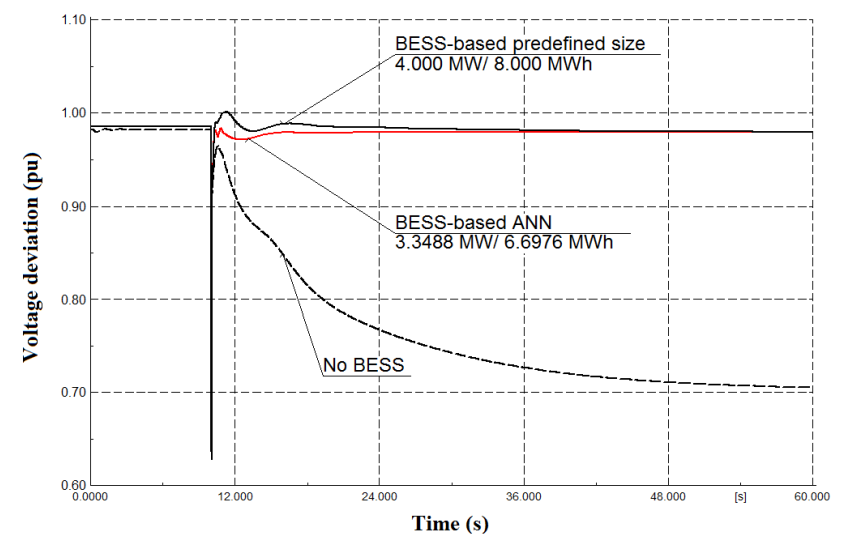

Fig. 8. Voltage deviation of the microgrid after islanding.

\section{Conclusion}

The accurate evaluation of the BESS size is critically important for the microgrid system when the microgrid is isolated from the main grid. An available BESS size computation model based on the Artificial Neural Network (ANN) has been proposed. The accuracy of the proposed neural network has been verified by using the measured data set. Based on the results, the comparison between the estimated results and the measured data demonstrate a very good agreement, hence verifying the effectiveness and accuracy of the proposed neural network (See Fig. 5 and Fig. 6). It can be conclude that the optimal size of BESS-based ANN gives better performance the optimal size of BESS-based predefined size (See Fig. 7 and Fig. 8). In addition, this approach does not depend on the physics of BESS, since the artificial neural network is a data-driven approach. Based on the result, the proposed neural network can be readily applied to BESS with different types and technologies. 


\section{References}

[1] Nick M, Cherkaoui R, Paolone M. Optimal siting and sizing of distributed energy storage systems via alternating direction method of multipliers. Electrical Power and Energy Systems, March 2015; 92(2015):33-39.

[2] Lamadrid AJ. Optimal use of energy storage systems with renewable energy sources. Electrical Power and Energy Systems, 2015; 71:101-111.

[3] Sigrist L, Lobato E, Rouco L. Energy storage systems providing primary reserve and peak shaving in small isolated power systems: An economic assessment. Electrical Power and Energy Systems, 2013; 53:675-683.

[4] Lin XN, Sun JW, Ai SF, Xiong XP, Wanc YF, Yang DX. Distribution network planning integrating charging stations of electric vehicle with V2G. Electrical Power and Energy Systems, 2014; 63:507-512.

[5] Dharmakeerthi CH, Mithulananthan N, Saha TK. Impact of electric vehicle fast charging on power system voltage stability. Electrical Power and Energy Systems, 2014; 57:241-249.

[6] Ali R, Hassan Mohamed T, Soliman Qudaih Y, Mitani Y. A new load frequency control approach in an isolated small power systems using coefficient diagram method. Electrical Power and Energy Systems, 2014; 56:110-116.

[7] Jamian JJ, Mustafa MW, Mokhlis H, Baharudin MA. Simulation study on optimal placement and sizing of battery switching station units using artificial bee colony algorithm. Electrical Power and Energy Systems, 2014; 55:592-601.

[8] Qudaih YS, Mitani Y. Power distribution system planning for smart grid applications using ANN. Energy Procedia, 2011; 12:3-9.

[9] Kerdphol T, Qudaih Y, Mitani Y. Battery energy storage system size optimization in microgrid using particle swarm optimization. In: Proc. IEEE PES Innovative Smart Grid Technologies Conference Europe Istanbul, Turkey, Oct. 2014.

[10] Jabalameli N, Masoum MAS. Battery storage unit for residential rooftop PV system to compensate impacts of solar variations. Electrical and Electronics Engineering: An International Journal, November 2013; 2(4).

[11] Balato M, Vitelli M. A new control strategy for the optimization of Distributed MPPT in PV applications. International Journal of Electrical Power \& Energy Systems, November 2014; 62:763-773. 